\title{
STUDYING THE GALACTIC CENTRAL ENGINE FROM SPACE OBSERVATORIES
}

\author{
HOWARD D. GREYBER \\ Greyber Associates, 10123 Falls Road, Potomac, MD 20854, U.S.A.
}

\section{Introduction}

Three general models have been constructed for the fantastically powerful "central engine" that powers the enormous energy output from quasars and active galactic nuclei (AGN). One model assumes a rapidly rotating accretion disk around a central black hole (however the disks, thick or thin, are subject to violent instabilities). Another assumes that in some postulated circuitry energy is extracted from the rotational portion of the deepest potential hole known, a black hole. Both models appear implausible.

The third model is the STRONG MAGNETIC FIELD MODEL (SMF) in which an extremely strong gravitationally bound current loop (GBCL) is formed during the gravitational collapse that forms the galaxy or quasar, producing a very intense dipole magnetic field anchored in the nucleus. SMF, first published in 1962, thus predicted the vertical magnetic field configuration seen today at our own galactic nucleus; to some the radio arcs observed suggest a dipole magnetic field there, just as SMF predicts.

When accretion occurs the diamagnetic effect of the bulk plasma produces topological magnetic mirrors as seen in Figure 1. The explanation for K. Kellerman's 1985 comment, "Typical morphology shows a compact core with one or more blobs moving away in a single direction" is simply that the throat must open when the plasma pressure locally exceeds the magnetic field pressure, so that a radio blob is expelled. The succession of blobs produced as accretion continues constitutes the radio jets observed.

SMF does not assume equipartition and thus does not need the exotic acceleration mechanisms required by other models. The two other models choose rotation as the important fundamental axial vector. SMF is different, choosing magnetic field.

In SMF the general morphology, energy production and dynamics of objects of galactic dimension are determined by the ratio of magnetic field energy to rotational energy. This ratio is extremely high for quasars and BL Lac objects, then decreasing as one goes to radio galaxies, Seyferts, Markarians, ordinary spirals, until it is close to zero for the ordinary elliptical galaxies. However, the activity seen is a function of the matter accretion rate at the time of observation! A great number of "quiet engines" may exist in the Universe.

(C) 1990 Kluwer Academic Publishers. Printed in The Netherlands. 


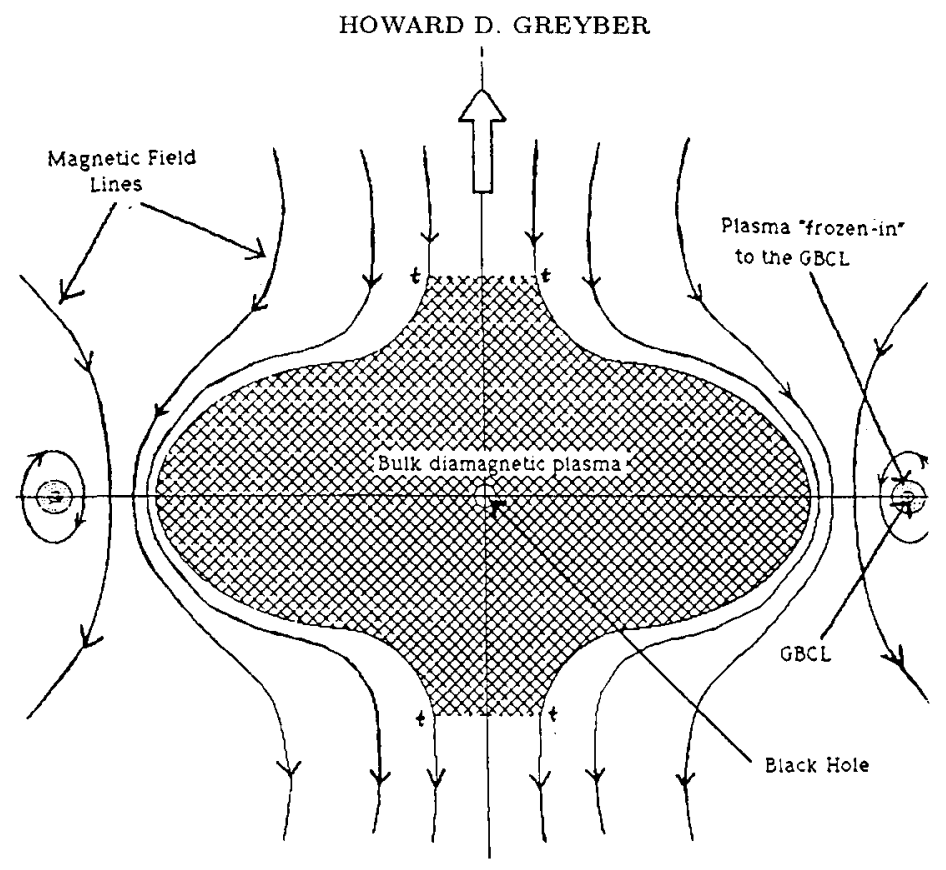

Fig. 1. SMF model for a quasar, BL Lac object or AGN when a very large accretion of matter around the black hole has occurred. $t-t$ is the throat of the magnetic mirrors. The arrow shows the direction of the motion of the explosively ejected hot plasma blob after the upper throat suddenly opens. As accretion continues, the process repeats every few years.

\section{Suggested Crucial Observations}

Since, unlike other models, SMF, predicts magnetic fields of 10,000 megagauss and higher at the center of galaxies and quasars, it would be desirable to measure the Zeeman splitting of some line. This may be easier from space observatories. SMF predicts that with better radio resolution (below the microarcsecond level), possibly gained from coupling an antenna in space to the VLBI networks on Earth, one should find evidence for a relatively weak GBCL around Sag $\mathrm{A}^{*}$, which is at the center of our Milky Way galaxy. An infrared interferometer located in space or on the Moon would also aid in the detection of a GBCL. Other evidence for the presence of a GBCL would be rapid brightness changes, or rapid changes of polarization intensity and/or direction in narrow regions located symmetrically on each side of the central object.

Another method is to search for rapid fluctuations in intensity or polarization from the quasar core or AGN. Several fluctuations of the order of 100 seconds or less have already been reported. This is significant since black hole masses greater than 100 million solar masses are required to power the strongest radio sources - and such a black hole has a diameter of 2000 light-seconds. If space observations confirm such rapid time variations, other models apparently must be rejected. However in SMF very rapid time variations can occur as neutral matter accretes and plunges across 
the minor diameter of the thin GBCL toroid, ionizes and radiates in the intense magnetic field close to the loop. In SMF it is not the quasar diameter that limits the characteristic time for a significant intensity variation, but the small diameter of the extremely slender GBCL.

Other crucial observations are to verify from space observations important conclusions about galaxies and quasars drawn from hundreds of papers of mostly ground-based observations.

Another crucial test is to analyze the magnetic field configuration in spiral arms close to the galactic nucleus. SMF predicts the field lies in one direction along the spiral arm above the plane of the galaxy, and in the other direction below the plane. with a neutral magnetic field sheet delineating the plane.

\section{Conclusions}

The expanded use of space observatories in all wavelength ranges should enable us to understand finally the physics of the "central engine" that powers the enormous energy output we observe from quasars and AGN. This physics will probably also explain the formation of galaxies and the variety of objects of galactic dimension that are observed.

\section{References}

Greyber, H.D., 1990 Strong Magnetic Fields, Galaxy Formation and the Galactic Engine in 14th Texas Symposium on Relativistic Astrophysics, New York: Annual of the New York Acad. of Sciences, $\mathbf{5 7 1} 239$, and earlier references cited therein.

\begin{tabular}{l|l|l} 
TABLE 1: COMPARISON OF THE STRONG MAGNETIC FIELD MODEL (SMF) \\
WITH THE ROTATING ACCRETION DISK MODEL
\end{tabular}

